Tohoku J. Exp. Med., 2008, 214, 165

\title{
Editorial
}

\section{Beauty in the Architecture of Living Organisms}

The Tohoku Journal of Experimental Medicine (TJEM) was founded in 1920. Since then, the TJEM is published continuously, except for the year of 1946 just after the World War II. The TJEM publishes original research papers that make novel and important contributions to the understanding of any area of medical sciences. In 2004, Tohoku University Medical Press, a nonprofit publisher of the TJEM, also launched the TJEM as a free online journal; namely, all articles published in the TJEM (from 1981) are accessible through PubMed. In fact, there were 141,343 article downloads in 2007. A total of 514 referees worldwide participated in peer review of selected manuscripts in 2007. More information is available at the website of the TJEM (http://journal. med.tohoku.ac.jp/).

One of the important papers published in the TJEM was by Ototaka Higashi, who observed the enlarged peroxidase granules in blood leukocytes derived from a patient with pigmentary abnormality and recurrent infections [HIGASHI, O. Congenital Gigantism of Peroxidase Granules. (1954) Tohoku J. Exp. Med., 59, 315-332]. His observation contributed to establishment of the new disease concept, now known as Chediak-Higashi syndrome. Indeed, seeing cells is invaluable in the field of medicine for understanding the complex interactions among different cell types. To enjoy the beauty in the architecture of living organisms, we need the good communication with histologists. However, the interactions between histologists and basic scientists with other specialties, such as molecular biologists or physiologists, are generally limited.

I, as the Editor-in-Chief, am pleased to orga- nize this issue to highlight the scientific achievement of Professor Hisatake Kondo, Department of Histology, Tohoku University School of Medicine. In commemoration of his retirement from the Professor position on March 31, 2008, his seven disciples, who have grown up from the Kondo's laboratory to become Professors in medical or dental school, attempt to publish a collection of papers: four review articles from Masahiko Watanabe, Kaoru Goto, Yuji Owada, and Hiroyuki Sakagami, and three original articles from Shoichi Iseki, Hiroshi Abe, and Osamu Amano. The review articles deal with selected molecules involved in the intracellular signaling in the brain. H. Kondo also contributes to this collection with his own review article as a piece of the series of his life-long study on the embedment-free section electron microscopy, in which he has been involved for these past 25 years. I congratulate on his happy retirement.

The latter part of this issue contains three original reports that are regular contributions. Incidentally, the report from Hiroyuki Takano and his colleagues shows the important role of estrogen in fine-tuning of growth potential of growth plate chondrocytes, with demonstrable sets of morphological data (page 269 of this issue).

I hope that all the readers of the TJEM appreciate the scientific principle of this special issue and that scientists will benefit from good collaboration with histologists.

Shigeki Shibahara

Editor-in-Chief

Tohoku Journal of Experimental Medicine 